\title{
DETERMINAÇÃO EXPERIMENTAL E MODELAGEM TERMODINÂMICA DO SISTEMA AQUOSO BIFÁSICO POLIETILENO GLICOL 4000, SULFATO DE LÍTIO E ÁGUA A $35^{\circ} \mathrm{C}$
}

\author{
R. K. FERREIRA ${ }^{1}$, L. S. LIMA ${ }^{1}$ e G. R. dos SANTOS ${ }^{2}$ \\ ${ }^{1}$ Universidade Federal do Pará, Faculdade de Engenharia Química \\ ${ }^{2}$ Universidade Federal do Pará, Faculdade de Engenharia de Alimentos \\ E-mail para contato: ramonkleyton15@hotmail.com
}

\begin{abstract}
RESUMO - Neste trabalho foi realizado o estudo do equilíbrio líquido-líquido do sistema aquoso bifásico composto por polietileno glicol de massa molecular média de $4000 \mathrm{~g} / \mathrm{mol}$ (PEG 4000), sulfato de lítio e água a $35^{\circ} \mathrm{C}$. Para isso, foi construída a curva binodal do sistema (curva de solubilidade) e a determinação de dados de equilíbrio do sistema. A quantificação dos componentes em cada fase nas células de equilíbrio foi realizada de forma indireta com o uso de dados da curva binodal, curvas de calibração com medidas de condutividade e resolução de um sistema de equações. Também foi realizada a modelagem termodinâmica do equilíbrio de fases do sistema utilizando o modelo NRTL, o qual conseguiu representar os dados do sistema proposto com desvio de $0,93 \%$ entre os dados experimentais e calculados.
\end{abstract}

\section{INTRODUÇÃO}

Sistemas aquosos bifásicos (SABs) têm adquirido importância e crescente sucesso como ferramenta na partição e/ou concentração de compostos como células animais ou vegetais, microorganismos, fungos e seus esporos, cloroplastos, ácidos nucleicos, vírus, metais e principalmente proteínas e enzimas. De acordo com Cunha (2008), os SABs são formados tradicionalmente pela mistura de dois diferentes polímeros ou de um polímero e um sal em água, que se separam em duas fases, ambas com alta concentração de água, com condições pouco agressivas, sem degradar ou prejudicar a biomolécula de interesse.

Diante desta área promissora, diversos pesquisadores têm desenvolvido trabalhos com sistemas aquosos bifásicos envolvendo polímero e sal, visto que informações sobre o diagrama de fases, bem como o desenvolvimento da modelagem termodinâmica do equilíbrio de fases são pontos importantes para o desenvolvimento, projeto, simulação e operação desses processos de separação. A exemplo disto têm-se os trabalhos de Graber et al. (2004) e Carvalho et al (2007), os quais trabalharam com o sistema PEG 4000, sulfato de lítio e água a várias temperaturas e Alvarenga et al.(2014) que mediram e correlacionaram os dados de equilíbrio de sistemas formados por PEG 4000 ou 1500, sulfato de sódio e água, com os modelos NRTL e UNIQUAQ. Sendo assim, o objetivo deste trabalho é determinar dados do equilíbrio do sistema aquoso bifásico formado por polietileno glicol de massa molecular 
média de $4000 \mathrm{~g} / \mathrm{mol}$ (PEG 4000), sulfato de lítio e água a $35^{\circ} \mathrm{C}$, assim como também realizar a modelagem termodinâmica utilizando o modelo NRTL.

\section{MATERIAIS E MÉTODOS}

\subsection{Materiais}

Para a obtenção dos dados de equilíbrio líquido-líquido do sistema proposto, foram utilizados polietileno glicol (PEG) de massa molecular média de $4000 \mathrm{~g} / \mathrm{mol}$ (Vetec, Brasil), sulfato de lítio anidro P.A a 98\% (Vetec, Brasil) e água destilada.

\subsection{Curva Binodal}

O procedimento de construção da curva binodal foi realizado conforme metodologia descrita por Azevedo (2015), na qual partem-se de soluções estoques de PEG e Sal a uma determinada porcentagem mássica. Dependendo da região da curva binodal a ser construída, acrescenta-se PEG à solução de sal ou sal à solução de PEG até a separação de fases. Em seguida, titula-se o sistema de duas fases com água destilada até ser atingida a transição entre o sistema bifásico e monofásico. Este ponto é caracterizado pela turvação do sistema, sendo também chamado de cloudy point.

Utilizaram-se neste trabalho soluções estoques de PEG 4000 a $50 \%$ (m/m) e sulfato de lítio a $25 \%(\mathrm{~m} / \mathrm{m})$. Os pontos da curva binodal foram obtidos a temperatura de $35^{\circ}$. Para isso foi utilizado um banho ultratemostático (Quimis, $\pm 0,1^{\circ} \mathrm{C}$ ). A quantificação dos componentes foi feita através de método gravimétrico na qual foi utilizada uma balança analítica (Sartoriuos TE214S, $\pm 0,0001 \mathrm{~g}$ ).

\subsection{Dados de Equilíbrio Líquido-Líquido}

Após a determinação da curva de miscibilidade parcial, curva binodal, soluções de composição conhecida e dentro da região de duas fases foram preparadas em tubos de ensaio, os quais funcionaram como células de equilíbrio. As soluções dentro das células foram agitadas por um período de 10 minutos para promover completa homogeneização do sistema. Após o período de agitação, as células foram mantidas em repouso por 24 horas a $35^{\circ} \mathrm{C}$ para que ocorresse a completa separação de fases. Para isso, as células de equilíbrio foram imersas em um banho termostático (Thermomix BM, $\pm 0,1^{\circ} \mathrm{C}$ ).

A quantificação dos componentes nas fases superior e inferior foi determinada por método indireto através da resolução de um sistema de equações, método similar ao realizado por Azevedo (2014). Neste sistema, as frações mássicas de PEG 4000, sulfato de lítio e água são as variáveis, sendo, desta forma, necessárias três equações para a sua resolução. A primeira equação foi obtida através da medida de condutividade elétrica (Medidor de Bancada Multiparâmetro, EDGE, $\pm 0,01 \mathrm{mS} / \mathrm{cm})$, a Equação 1 mostra de forma representativa a condutividade elétrica em função das composições de PEG 4000 e sulfato de lítio. A segunda equação do sistema foi obtida da correlação dos dados da curva binodal, obtendo-se uma equação da composição de PEG 4000 em função da composição de sulfato de lítio, a Equação 2 mostra de forma representativa esta função. A terceira equação do sistema representa a soma das frações mássicas, conforme a Equação 3. Com a resolução deste sistema, têm-se 
resultados com a precisão de $\pm 0,01$ para a fração mássica de PEG 4000 e $\pm 0,0009$ para a fração de mássica de sulfato de lítio. A seguir são mostradas, de forma representativa, as equações utilizadas para a resolução do sistema matemático, onde $x_{P E G}, x_{L i 2 S O 4}$ e $x_{\text {água }}$ são, respectivamente, as frações mássicas de PEG 4000, sulfato de lítio e água.

$$
\begin{aligned}
& \text { Condutividade }=f\left(x_{P E G}, x_{\mathrm{Li}_{2} \mathrm{SO}_{4}}\right) \\
& X_{P E G}=f\left(x_{\mathrm{Li}_{2} \mathrm{SO}_{4}}\right) \\
& x_{P E G}+x_{\mathrm{Li2} 2 \mathrm{SO}_{4}}+x_{\text {Agua }}=1
\end{aligned}
$$

\subsection{Modelagem Termodinâmica}

Após a determinação dos dados de equilíbrio foi realizada a modelagem termodinâmica dos dados. A estimativa dos parâmetros de interação energética entre as moléculas de PEG 4000, sulfato de lítio e água para o modelo NRTL (Non Random, TwoLiquid) (Renon e Prausnitz, 1968), foram executadas utilizado o programa TML-LLE; o procedimento é baseado no método simplex modificado (Nelder e Mead, 1965) e consiste na minimização de uma função objetivo, S, baseada na concentração, conforme mostra a Equação 4.

$$
\left.S=\sum_{k}^{D} \sum_{j}^{M} \sum_{i}^{N-1}\left(x_{i j k}^{I, \exp }-x_{i j k}^{I, \text { calc }}\right)^{2}+\left(x_{i j k}^{I I, \exp }-x_{i j k}^{I I, \text { calc }}\right)^{2}\right)
$$

$\mathrm{Na}$ equação anterior, $\mathrm{D}$ é o número de conjunto de dados, $\mathrm{N}$ e $\mathrm{M}$ são os números de componentes e linhas de amarração em cada conjunto de dados, respectivamente; os sobrescritos I e II representam as duas fases líquidas em equilíbrio, enquanto os sobrescritos 'exp' e 'calc' referem-se aos valores experimentais e calculados das concentrações das fases.

Com os parâmetros calculados pelo procedimento acima, os dados de equilíbrio líquidolíquido são correlacionados executando um cálculo de flash líquido-líquido. Comparações entre as composições experimentais e calculadas para cada componente das duas fases são feitas por meio do desvio quadrado médio, dado pela Equação 5.

$$
\delta x=100 \sqrt{\frac{\sum_{i}^{M} \sum_{j}^{N-1}\left(x_{i j}^{I, e x p}-x_{i j}^{I, c a l c}\right)^{j}+\left(x_{i j}^{I I, e x p}-x_{i j}^{I I, c a l c}\right)^{2}}{2 M N}}
$$

\section{RESULTADOS E DISCUSSÕES}

\subsection{Curva Binodal e Curva de Calibração}

Através da regressão de dados da curva binodal, foi possível obter um modelo exponencial que representasse o seu comportamento com um $\mathrm{R}^{2}=0,9959$. A Equação 7 mostra o modelo ajustado para a curva binodal, assim como a Figura 1 mostra a comparação da curva experimental a $35^{\circ} \mathrm{C}$ com o modelo ajustado. 


$$
\left.x_{P E G}=E X P^{\left(-0,4535-11,7536 x_{L i 2 S O 4}-137,1347 x_{L i 2 S O 4}^{2}\right.}\right)
$$

Figura 1 - Curva binodal $(\bullet)$ e seu modelo representativo $(-)$.

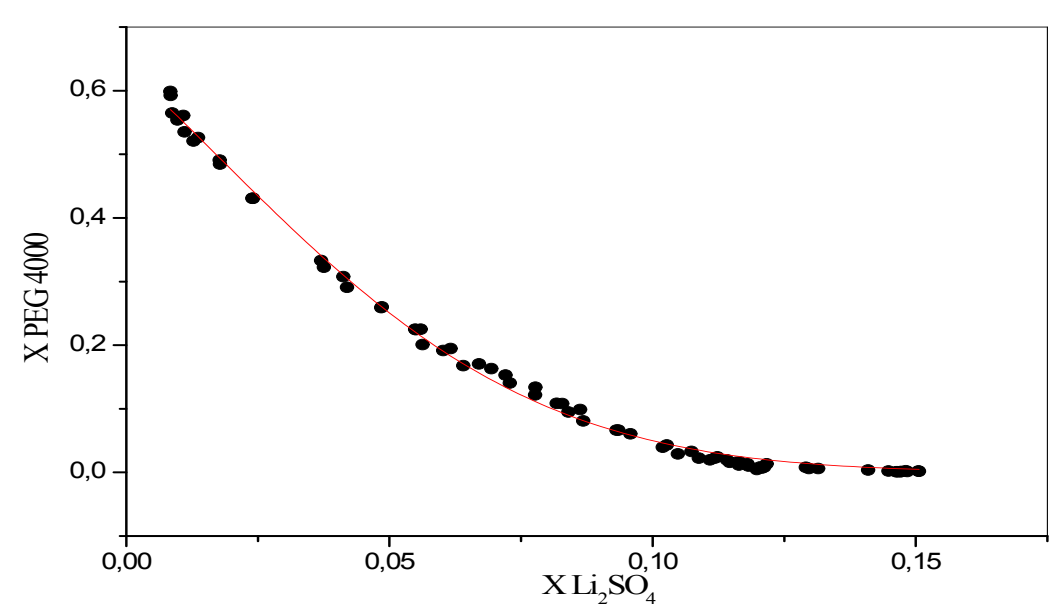

Com os dados da condutividade elétrica da curva de calibração, também pela regressão de dados, foi possível obter um modelo parabólico que representasse o comportamento da condutividade elétrica em função das composições de PEG 4000 e sulfato de lítio com um $\mathrm{R}^{2}$ $=0,9994$, assim como mostra a Equação 8 .

$$
\text { Condutividade }=3,0437-4,5880 x_{P E G}+12,4179 x_{L i 2 S O 4}+0,2229 x_{P E G}^{2}+153,0887 x_{L i 2 S O 4}^{2}
$$

\subsection{Linhas de Amarração Experimentais e Modelagem Termodinâmica}

As concentrações das fases, superior e inferior, de cada célula de equilíbrio foram determinadas através da solução do sistema de equações formado pelas Equações 3, 7 e 8 . A Tabela 1 mostra a composição global e as composições da fase superior e inferior de cada célula de equilíbrio.

Tabela 1 - Composição das células de equilíbrio

\begin{tabular}{c|ccc|ccc|ccc}
\hline \multicolumn{4}{c}{ Global } & \multicolumn{3}{c}{ Fase Superior } & \multicolumn{3}{c}{ Fase Inferior } \\
\hline Linha & $x_{P E G}$ & $x_{\text {Li2SO4 }}$ & $x_{\text {Agua }}$ & $x_{P E G}$ & $x_{\text {Li2SO4 }}$ & $x_{\text {Agua }}$ & $x_{P E G}$ & $x_{\text {Li2SO4 }}$ & $x_{\text {Agua }}$ \\
\hline 1 & 0,2248 & 0,0619 & 0,7133 & 0,3103 & 0,0415 & 0,6482 & 0,0166 & 0,1271 & 0,8563 \\
2 & 0,2491 & 0,0653 & 0,6856 & 0,3622 & 0,0344 & 0,6034 & 0,0099 & 0,1381 & 0,8520 \\
3 & 0,2770 & 0,0702 & 0,6529 & 0,4261 & 0,0262 & 0,5477 & 0,0051 & 0,1514 & 0,8435 \\
4 & 0,2595 & 0,0679 & 0,6726 & 0,4118 & 0,0280 & 0,5602 & 0,0071 & 0,1448 & 0,8481 \\
5 & 0,2223 & 0,0610 & 0,7168 & 0,2846 & 0,0452 & 0,6702 & 0,0284 & 0,1149 & 0,8567 \\
6 & 0,2796 & 0,0709 & 0,6495 & 0,4464 & 0,0237 & 0,5299 & 0,0039 & 0,1562 & 0,8399 \\
\hline
\end{tabular}

A Figura 2 mostra os dados de equilíbrio líquido-líquido representado pela curva binodal e pelas linhas de amarração experimentais. 
Figura 2 - Dados de equilíbrio líquido-líquido: curva binodal (•) e linhas de amarração experimentais $(\square)$.

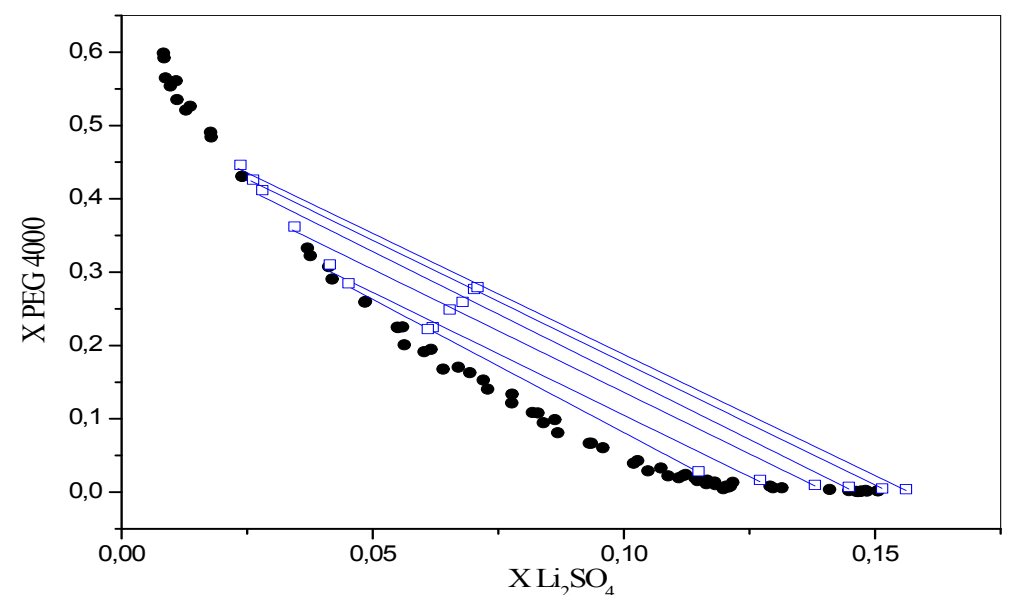

A modelagem termodinâmica feita através do modelo NRTL obteve um desvio quadrado médio de $0,9288 \%$ entre os valores estimados pelo modelo e os valores experimentais. A Figura 3 mostra a comparação entre as linhas de amarração obtidas experimentalmente e as estimadas pelo modelo. A Tabela 2 mostra os parâmetros de interação binária $\left(\tau_{\mathrm{ij}} \mathrm{e} \tau_{\mathrm{ji}}\right)$ e não-aleatoriedade $(\alpha)$, do modelo NRTL, obtidos através da modelagem.

Figura 3 - Linhas experimentais (•) e linhas estimadas pelo modelo NRTL ( $\square$ ).

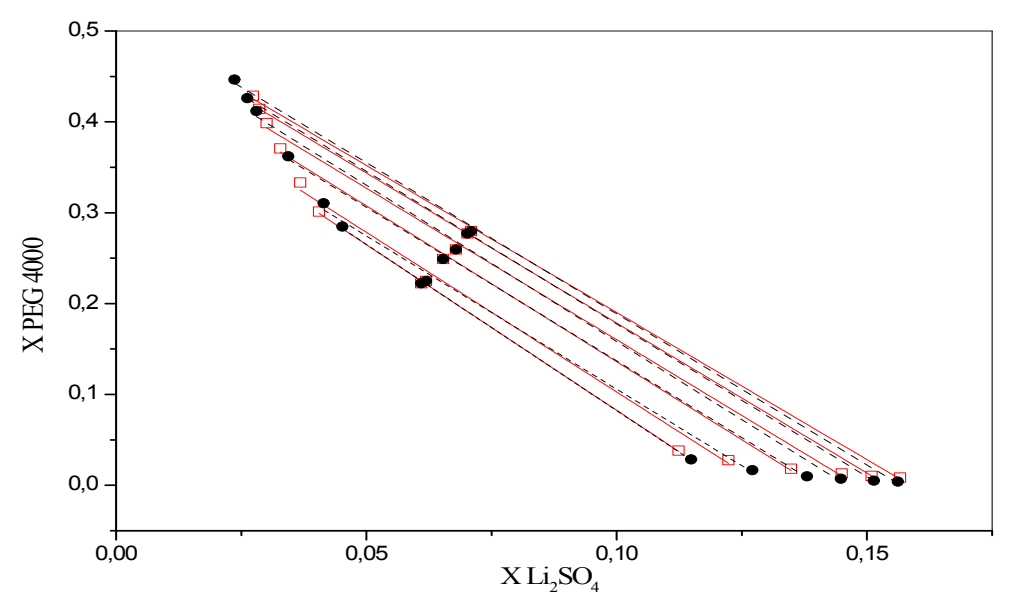

Tabela 2 - Parâmetros ajustados do modelo NRTL

\begin{tabular}{c|ccc}
\hline $\mathrm{i}-\mathrm{j}$ & $\tau_{\mathrm{ij}}$ & $\tau_{\mathrm{ji}}$ & $\alpha$ \\
\hline $\mathrm{PEG}-\mathrm{Li}_{2} \mathrm{SO}_{4}$ & $-0,53425$ & 8451,6 & 0,20905 \\
\hline $\mathrm{PEG}-$ Água & $-3568,3$ & 5031,6 & 0,26731 \\
\hline $\mathrm{Li}_{2} \mathrm{SO}_{4}-$ Água & $-1319,8$ & -9000 & 0,36828 \\
\hline
\end{tabular}




\section{CONCLUSÕES}

Dados experimentais de equilíbrio de sistema aquoso bifasico ternário PEG 4000, sulfato de lítio e água a $35^{\circ} \mathrm{C}$ foram determinados e quantificados usando dados da curva binodal e de curvas de calibração em função da condutividade. A curva binodal obtida apresentou um par de componentes miscíveis e foi descrita por uma equação exponencial e apresentou um coeficiente de correlação de 0,9959. A curva de calibração foi descrita por uma equação de segunda ordem com coeficiente de correlação próximo a um $\left(\mathrm{R}^{2}=0,9994\right)$. A precisão das medidas experimentais foi de $\pm 0,01$ para o PEG 4000 e $\pm 0,0009$ para o sulfato de lítio. O modelo NTRL foi utilizado para correlacionar dados experimentais obtidos e obteve-se um desvio médio quadrático entre as composições calculadas e experimentais em ambas as fases de equilíbrio de $0,93 \%$.

\section{REFERÊNCIAS}

ALVARENGA, B. G.; VIRTUOSO, L, S.; LEMES, N, H,T.; SILVA, L, A. da.; MESQUITA, A, F.; NASCIMENTO, K, S.; SILVA, M, C, HESPANHOL, da.; SILVA, L, H, M, da. Meassurement and Correlation of the Phase Equilibrium of Aqueous Two-Phase Systems Composed of Polyethylene(glycol) 1500 or $4000+$ Sodium Sulfite + Water at Different temperatures. Journal Of Chemical \& Engineering Data, v. 59, p.382-390, 5 fev. 2014.

AZEVEDO, C, M. DETERMINAÇÃO EXPERIMENTAL E MODELAGEM TERMODINÂMICA DO SISTEMA EM DUAS FASES AQUOSAS POLIETILENO GLICOL, SULFATO DE LÍTIO A 303,15 K. 2015. 39 f. TCC (Graduação) - Curso de Engenharia Química, Instituto de Tecnologia, Universidade Federal do Pará, Belém, 2015.

CARVALHO, C, P.; COIMBRA, J, S, R.; COSTA, I, A, F.; MINIM, L, A.; SILVA, L, H, M.; MAFFIA, M, C. Equilibrium Data for PEG $4000+$ Salt + Water Systems from $(278.15$ to 318.15) K. Journal Of Chemical \& Engineering Data, Belem, v. 52, p.351-356, 2 jun. 2007.

CUNHA, E, V, C. EQUILÍBRIO LÍQUIDO-LÍQUIDO EM SISTEMAS AQUOSOS BIFÁSICOS ÁGUA + PEG 8000 + SAL: DETERMINAÇÃO EXPERIMENTAL E MODELAGEM TERMODINÂMICA. 2008. 94 f. Dissertação (Mestrado) - Curso de Engenharia Química, Faculdade de Engenharia Química, Universidade Estadual de Campinas, Campinas, 2008.

GRABER, T, A.; GÁlveZ, M, E.; GALlEGUILlos.; BENEDÍ, J, A. Liquid-Liquid Equilibrium of Aqueous Two-Phase System Water + PEG $4000+$ Lithium Sulfate at Different Temperatures.: Experimental Determination and Correlation. Journal of Chemical \& Engineering Data, Belem, v. 49, p.1661-1664, 9 fev. 2004.

NELDER, J, A.; MEAD, R. A Simplex Method for Function Minimization. The Computer Journal, v. 7, p.308-313, 1965.

RENON, H.; PRAUSNITZ, J, M. Local Compositions in Thermodynamic Excess Functions for Liquid Mixtures. AlChe Journal, v. 14, p.135-144, jan. 1968. 